\title{
HIMPUNAN KUBIK ASIKLIK DAN KUBUS DASAR
}

\author{
WIWI ULMAYANI \\ Program Studi Matematika, \\ Fakultas Matematika dan Ilmu Pengetahuan Alam, Universitas Andalas, \\ Kampus UNAND Limau Manis Padang, Indonesia, \\ wiwi.ulmayani@ymail.com
}

\begin{abstract}
Given a topological space $X$. Then define an algebra object $H_{*}(X)$ which is called the homology group of $\mathrm{X} . H_{*}(X)$ is the collection the $k^{\text {th }}$ homology group of $X$ which is denoted by $H_{k}(X)$. An elementary cube $Q$ is a finite product of elementary intervals $I=[l, l+1]$ or $I=[l, l]$, for some $l \in \mathbb{Z}$. In this paper, it is proved that all elementary cubes are acyclic, which means that $H_{k}(Q)$ is isomorphic to $\mathbb{Z}$ if $k=0$, and $H_{k}(Q)$ is isomorphic to 0 if $k>0$.
\end{abstract}

Kata Kunci: Topological space, acyclic, isomorphic

\section{Pendahuluan}

Misalkan diberikan ruang topologi $X$. Selanjutnya didefinisikan suatu objek aljabar $H_{*}(X)$ yang disebut dengan homologi dari $X$, dimana secara topologi $H_{*}(X)$ adalah sebuah invarian, artinya jika $X$ dan $Y$ adalah homeomorfik maka $H_{*}(X)$ dan $H_{*}(Y)$ adalah isomorfik,

$$
X \approx Y \Rightarrow H_{*}(X) \cong H_{*}(Y),
$$

dimana $H_{*}(X)$ merupakan koleksi dari grup homologi ke-k dari $X$ yang dinotasikan dengan $H_{k}(X)$.

Misalkan diberikan suatu ruang topologi $G \subset \mathbb{R}^{n}$, yang mana dapat disederhanakan menjadi suatu graf. Kemudian graf tersebut diobservasi dan direpresentasikan secara kombinatorik dan dari kombinatorik ini diperoleh suatu objek aljabar $H_{*}(G)$ yang disebut homologi dari $G$.

Pada tulisan ini, penulis akan fokus pada homologi kubik dimana ruang topologi dapat direpresentasikan sebagai sebuah kubik. Sebuah kubus dasar $Q$ adalah suatu hasil kali hingga dari interval-interval dasar $I=[l, l+1]$ atau $I=[l, l]$ untuk suatu $l \in \mathbb{Z}$. Jadi, $Q=I_{1} \times I_{2} \times \cdots \times I_{n} \subset \mathbb{R}^{n}$. Himpunan kubik merupakan suatu kelas khusus dari ruang topologi.

Berdasarkan definisi, sebuah himpunan kubik $X$ disebut asiklik jika

$$
H_{k}(X) \cong\left\{\begin{array}{l}
\mathbb{Z}, \text { jika } k=0 \\
0, \text { selainnya }
\end{array}\right.
$$

untuk $k \geq 0$ dan $k \in \mathbb{Z}$.

Makalah ini bertujuan untuk mengkaji hubungan antara himpunan kubik asiklik dan kubus dasar. 
44 Wiwi Ulmayani

\section{Himpunan Kubik Asiklik dan Kubus Dasar}

Berikut akan diberikan definisi tentang himpunan kubik asiklik.

Definisi 2.1. [5] Suatu himpunan kubik $X$ dikatakan asiklik jika

$$
H_{k}(X) \cong\left\{\begin{array}{l}
\mathbb{Z}, \text { jika } k=0 \\
0, \text { selainnya }
\end{array}\right.
$$

untuk $k \geq 0$ dan $k \in \mathbb{Z}$.

Misalkan $Q=I_{1} \times I_{2} \times \cdots \times I_{d}$ sebuah kubus dasar. Untuk suatu $i \in\{1,2, \cdots, d\}$ misalkan $I_{i}(Q)$ nondegenerate, dengan $I_{i}(Q)=\left[l_{i}, l_{i}+1\right]$, untuk suatu $l_{i} \in \mathbb{Z}$. Pilih $k>0$. Keluarga face berdimensi $k$ dari $Q$ dapat diuraikan sebagai berikut

$$
\mathcal{K}_{k}(Q)=\mathcal{K}_{k}([l], i) \cup \mathcal{K}_{k}([l, l+1], i) \cup \mathcal{K}_{k}([l+1], i),
$$

dimana $\mathcal{K}_{k}(\Delta, i):=\left\{P \in \mathcal{K}_{k}(Q) \mid I_{i}(P)=\Delta\right\}$, dengan $\Delta:=[l],[l, l+1],[l+1]$.

Berikut adalah contoh keluarga face berdimensi $k=2$.

Contoh 2.2. Misalkan $Q=[p, p+1] \times[l, l+1] \times[q]$. Maka

$\mathcal{K}_{1}(Q)=\{[p, p+1] \times[l] \times[q],[p] \times[l, l+1] \times[q],[p+1] \times[l, l+1] \times[q],[p, p+1] \times[l+1] \times[q]\}$ dan

$$
\mathcal{K}_{2}(Q)=\{[p, p+1] \times[l, l+1] \times q\},
$$

sehingga diperoleh

$$
\begin{aligned}
\mathcal{K}_{1}([l], 2) & =\{[p, p+1] \times[l] \times[q]\}, \\
\mathcal{K}_{1}([l, l+1], 2) & =\{[p] \times[l, l+1] \times[q],[p+1] \times[l, l+1] \times[q]\} \\
\mathcal{K}_{1}([l+1], 2) & =\{[p, p+1] \times[l+1] \times[q]\}
\end{aligned}
$$

dan

$$
\begin{aligned}
\mathcal{K}_{2}([l], 2) & =\emptyset, \\
\mathcal{K}_{2}([l, l+1], 2) & =\{[p, p+1] \times[l, l+1] \times[q]\} \\
\mathcal{K}_{2}([l+1], 2) & =\emptyset .
\end{aligned}
$$

Misalkan $z \in Z_{k}(Q)$ yang memiliki sifat bahwa $|z|$ tidak memuat kubus dasar dengan komponen $[l+1]$. Maka $|z|$ tidak memuat kubus dasar dengan komponen $[l, l+1]$.

Lema berikut digunakan untuk membuktikan Teorema 2.4.

Lema 2.3. [5] Asumsikan $Q \in \mathcal{K}^{d}$ dan $i \in\{1,2, \cdots, d\}$. Jika $z$ merupakan siklik $k e$-k pada $Q$ sedemikian sehingga $\langle z, \widehat{P}\rangle=0$ untuk setiap $P \in \mathcal{K}_{k}([l+1], i)$, maka $\langle z, \widehat{P}\rangle=0$ untuk setiap $P \in \mathcal{K}_{k}([l, l+1], i)$.

Bukti. Karena $z$ merupakan rantai pada $Q$, maka

$$
z=\sum_{P \in \mathcal{K}_{k}(Q)}\langle z, \widehat{P}\rangle \widehat{P} .
$$


Oleh karena itu, untuk sebarang $R \in \mathcal{K}_{k-1}(Q)$, berlaku

$$
\begin{aligned}
\langle\partial z, \widehat{R}\rangle & =\left\langle\partial\left(\sum_{P \in \mathcal{K}_{k}(Q)}\langle z, \widehat{P}\rangle \widehat{P}\right), \widehat{R}\right\rangle \\
& =\left\langle\sum_{P \in \mathcal{K}_{k}(Q)}\langle z, \widehat{P}\rangle \partial \widehat{P}, \widehat{R}\right\rangle \\
& =\sum_{P \in \mathcal{K}_{k}(Q)}\langle z, \widehat{P}\rangle\langle\partial \widehat{P}, \widehat{R}\rangle .
\end{aligned}
$$

Karena $z$ siklik, maka untuk sebarang $R \in \mathcal{K}_{k-1}(Q)$ berlaku

$$
\begin{aligned}
0= & \langle\partial z, \widehat{R}\rangle=\sum_{P \in \mathcal{K}_{k}([l], i)}\langle z, \widehat{P}\rangle\langle\partial \widehat{P}, \widehat{R}\rangle+\sum_{P \in \mathcal{K}_{k}([l, l+1], i)}\langle z, \widehat{P}\rangle\langle\partial \widehat{P}, \widehat{R}\rangle+ \\
& \sum_{P \in \mathcal{K}_{k}([l+1], i)}\langle z, \widehat{P}\rangle\langle\partial \widehat{P}, \widehat{R}\rangle
\end{aligned}
$$

Karena $\langle z, \widehat{P}\rangle=0$ untuk setiap $P \in \mathcal{K}_{k}([l+1], i)$, maka

$$
0=\langle\partial z, \widehat{R}\rangle=\sum_{P \in \mathcal{K}_{k}([l], i)}\langle z, \widehat{P}\rangle\langle\partial \widehat{P}, \widehat{R}\rangle+\sum_{P \in \mathcal{K}_{k}([l, l+1], i)}\langle z, \widehat{P}\rangle\langle\partial \widehat{P}, \widehat{R}\rangle
$$

Misalkan $P_{0} \in \mathcal{K}_{k}([l, l+1], i)$, dan misalkan $R_{0}$ kubus dasar yang didefinisikan sebagai

$$
I_{j}\left(R_{0}\right)=\left\{\begin{array}{l}
{[l+1], \text { jika } \mathrm{j}=\mathrm{i}} \\
I_{j}\left(P_{0}\right), \text { selainnya. }
\end{array}\right.
$$

Maka, $R_{0}$ bukan face dari $P$ untuk $P \in \mathcal{K}_{k}([l], i)$, karena $I_{i}(P)=[l]$ sedangkan $I_{i}\left(R_{0}\right)=[l+1]$. Akibatnya $\sum_{P \in \mathcal{K}_{k}([l], i)}\langle z, \widehat{P}\rangle\langle\partial \widehat{P}, \widehat{R}\rangle$ pada ruas kanan (2.3) tidak muncul untuk $R=R_{0}$. Namun, $R_{0}$ adalah face dari $P$ untuk $P \in \mathcal{K}_{k}([l, l+1], i)$ jika dan hanya jika $P=P_{0}$. Ini berarti bahwa persamaan (2.4) direduksi $R=R_{0}$ menjadi $0=\left\langle z, \widehat{P_{0}}\right\rangle\left\langle\partial \widehat{P_{0}}, \widehat{R}_{0}\right\rangle$.

Perhatikan bahwa

$$
\begin{aligned}
R_{0} & =I_{1} \times I_{2} \times \cdots \times I_{i} \times \cdots \times I_{d} \\
& =I_{1} \times I_{2} \times \cdots \times[l+1] \times \cdots \times I_{d} \\
\widehat{R_{0}} & =I_{1} \times I_{2} \times \cdots \times \widehat{[l+1]} \times \cdots \times I_{d} \\
& =\widehat{I_{1}} \diamond \widehat{I_{2}} \diamond \cdots \diamond \widehat{[l+1]} \diamond \cdots \diamond \widehat{I_{d}} \\
& =\widehat{[l+1]} \diamond \widehat{I_{1}} \diamond \widehat{I_{2}} \diamond \cdots \diamond \widehat{I_{i-1}} \diamond \widehat{I_{i+1}} \diamond \cdots \diamond \widehat{I_{d}} \\
& =\widehat{[l+1]} \diamond \underbrace{}_{\widehat{I_{1}} \diamond \widehat{I_{2}} \diamond \cdots \diamond \widehat{I_{i-1}} \diamond \widehat{I_{i+1}} \diamond \cdots \diamond \widehat{I_{d}}} \\
& =\widehat{[l+1]} \diamond \widehat{J^{\prime}}
\end{aligned}
$$


dan

$$
\begin{aligned}
P_{0} & =I_{1} \times I_{2} \times \cdots \times I_{i} \times \cdots \times I_{d} \\
& =I_{1} \times I_{2} \times \cdots \times[l, l+1] \times \cdots \times I_{d} \\
\widehat{P_{0}} & \left.=I_{1} \times I_{2} \times \cdots \times \widehat{[l, l}+1\right] \times \cdots \times I_{d} \\
& =\widehat{I_{1}} \diamond \widehat{I_{2}} \diamond \cdots \diamond[\widehat{l, l+1}] \diamond \cdots \diamond \widehat{I_{d}} \\
& =[\widehat{l, l+1}] \diamond \widehat{I_{1}} \diamond \widehat{I_{2}} \diamond \cdots \diamond \widehat{I_{i-1}} \diamond \widehat{I_{i+1}} \diamond \cdots \diamond \widehat{I_{d}} \\
& =\underbrace{[\widehat{l, l+1}]}_{\widehat{I^{\prime}}} \diamond \underbrace{\widehat{l}}_{\widehat{I_{1}} \diamond \widehat{I_{2}} \diamond \cdots \diamond \widehat{I_{i-1}} \diamond \widehat{I_{i+1}} \diamond \cdots \diamond \widehat{I_{d}}} .
\end{aligned}
$$

Sehingga,

$$
\begin{aligned}
\partial \widehat{P_{0}} & =\partial \widehat{I^{\prime}} \diamond \widehat{J^{\prime}}+(-1)^{\operatorname{dim} I^{\prime}} \widehat{I^{\prime} \diamond \partial \widehat{J^{\prime}}} \\
& =\partial[\widehat{l, l+1}] \diamond \widehat{J^{\prime}}+(-1)[\widehat{l, l+1}] \diamond \partial \widehat{J^{\prime}} \\
& =(\widehat{[l+1]}-\widehat{[l]}) \diamond \widehat{J^{\prime}}+(-1)[\widehat{l, l+1}] \diamond \partial \widehat{J^{\prime}} \\
& =\widehat{[l+1]} \diamond \widehat{J^{\prime}}-\widehat{[l]} \diamond \widehat{J^{\prime}}+(-1)[\widehat{l, l+1}] \diamond \partial \widehat{J}^{\prime} .
\end{aligned}
$$

Karena $\left\langle\partial \widehat{P_{0}}, \widehat{R}_{0}\right\rangle \neq 0$, diperoleh $\left\langle z, \widehat{P}_{0}\right\rangle=0$.

Teorema berikut merupakan hasil kajian utama dalam makalah ini.

Teorema 2.4. [5] Setiap kubus dasar adalah asiklik.

Bukti. Misalkan $Q$ adalah kubus dasar. Karena $Q$ connected, maka menurut [5], $H_{0}(Q) \cong \mathbb{Z}$. Sehingga cukup ditunjukkan bahwa $H_{k}(Q)=0$ untuk $k>0$, yang ekivalen dengan menunjukkan bahwa setiap siklik ke- $k$ pada $Q$ adalah batas (boundary). Dalam hal ini akan ditunjukkan dengan induksi pada $n:=\operatorname{dim} Q$.

- Untuk $n=0$.

Jika $n=0$, maka $Z_{k}(Q)=C_{k}(Q)=0=B_{k}(Q)$ yang menunjukkan bahwa $H_{k}(Q)=0$.

- Untuk $n>0$.

Asumsikan bahwa $H_{k}(Q)=0$, untuk semua kubus dasar dengan dimensi yang lebih kecil dari $n$. Karena $n>0$, pilih sebarang $i$ sedemikian sehingga $I_{i}(Q)$ nondegenerate. Untuk setiap $P \in \mathcal{K}_{k}([l+1], i)$, misalkan $P^{*}$ kubus dasar yang diberikan oleh

$$
I_{j}\left(P^{*}\right):= \begin{cases}{[l, l+1],} & \text { jika } j=i \\ I_{j}(P), & \text { selainnya. }\end{cases}
$$

Maka $P$ adalah face dari $P^{*}$. Misalkan $z$ sebuah siklik ke- $k$ pada $Q$. Definisikan

$$
\begin{aligned}
c & :=\sum_{P \in \mathcal{K}_{k}([l+1], i)}\langle z, \widehat{P}\rangle\left\langle\partial \widehat{P^{*}}, \widehat{P}\right\rangle \widehat{P^{*}} \\
z^{\prime} & :=z-\partial c
\end{aligned}
$$


Untuk setiap $P_{0} \in \mathcal{K}_{k}([l+1], i)$, berlaku

$$
\begin{aligned}
\left\langle\partial c, \widehat{P_{0}}\right\rangle & =\left\langle\partial\left(\sum_{P \in \mathcal{K}_{k}([l+1], i)}\langle z, \widehat{P}\rangle\left\langle\partial \widehat{P^{*}}, \widehat{P}\right\rangle \widehat{P^{*}}\right), \widehat{P_{0}}\right\rangle \\
& =\left\langle\sum_{P \in \mathcal{K}_{k}([l+1]}\langle z, \widehat{P}\rangle\left\langle\partial \widehat{P^{*}}, \widehat{P}\right\rangle \partial \widehat{P^{*}}, \widehat{P_{0}}\right\rangle \\
& =\sum_{P \in \mathcal{K}_{k}([l+1]}\langle z, \widehat{P}\rangle\left\langle\partial \widehat{P^{*}}, \widehat{P}\right\rangle\left\langle\partial \widehat{P^{*}}, \widehat{P_{0}}\right\rangle .
\end{aligned}
$$

Karena $I_{i}\left(P^{*}\right)=[l, l+1]$ dan $I_{i}\left(P_{0}\right)=[l+1]$, akibatnya $\left\langle\partial \widehat{P^{*}}, \widehat{P_{0}}\right\rangle \neq 0$ jika dan hanya jika $P=P_{0}$. Oleh karena itu, $\left\langle\partial c, \widehat{P_{0}}\right\rangle=\left\langle z, \widehat{P_{0}}\right\rangle$ dan $\left\langle z^{\prime}, \widehat{P_{0}}\right\rangle=0$. Berdasarkan Lema 2.3, $\left|z^{\prime}\right| \subset Q^{\prime}$, dimana $Q^{\prime}$ merupakan kubus berdimensi $n-1$ yang didefinisikan sebagai

$$
I_{j}\left(Q^{\prime}\right):= \begin{cases}{[l],} & \text { jika } j=i \\ I_{j}(Q), & \text { selainnya. }\end{cases}
$$

Dari induksi, diperoleh $z^{\prime}=\partial c^{\prime}$. Ini menunjukkan bahwa

$$
\begin{aligned}
z & =\partial c+z^{\prime}, \\
& =\partial c+\partial c^{\prime} \\
& =\partial\left(c+c^{\prime}\right),
\end{aligned}
$$

dengan $z$ adalah batas.

Kebalikan dari Teorema 2.4 tidak berlaku, karena setiap himpunan kubik yang asiklik belum tentu merupakan kubus dasar.

Contoh 2.5. Misal himpunan kubik

$$
\Gamma^{\prime}=[1,3] \times[2] .
$$

Definisikan $Q_{1}=[1,2] \times[2]$ dan $Q_{2}=[2,3] \times[2]$. Himpunan-himpunan dari kubus dasar $Q_{1}$ dan $Q_{2}$ adalah

$$
\begin{aligned}
& \mathcal{K}_{0}\left(Q_{1}\right)=[1] \times[2],[2] \times[2] \\
& \mathcal{K}_{0}\left(Q_{2}\right)=[2] \times[2],[3] \times[2] \\
& \mathcal{K}_{1}\left(Q_{1}\right)=[1,2] \times[2] \\
& \mathcal{K}_{1}\left(Q_{2}\right)=[2,3] \times[2]
\end{aligned}
$$

Maka basis untuk himpunan rantai-rantai dari $Q_{1}$ dan $Q_{2}$ adalah

$$
\begin{aligned}
\widehat{\mathcal{K}}_{0}\left(Q_{1}\right) & =\{[\widehat{[1] \times[2}], \widehat{[2] \times[2]}\} \\
& =\{\widehat{[1]} \diamond \widehat{[2]}, \widehat{[2]} \diamond \widehat{[2]}\}, \\
\widehat{\mathcal{K}}_{0}\left(Q_{2}\right) & =\{[\widehat{2] \times[2]}, \widehat{[3] \times[2]}\} \\
& =\{\widehat{[2]} \diamond \widehat{[2]}, \widehat{[3]} \diamond \widehat{[2]}\} .
\end{aligned}
$$




$$
\begin{aligned}
& \widehat{\mathcal{K}}_{1}\left(Q_{1}\right)=\{[1, \widehat{2] \times}[2]\} \\
& =\{\widehat{[1,2]} \diamond \widehat{[2]}\}, \\
& \widehat{\mathcal{K}}_{1}\left(Q_{2}\right)=\{[2, \widehat{3] \times}[2]\} \\
& =\{\widehat{[2,3]} \diamond \widehat{[2]}\} .
\end{aligned}
$$

Untuk menghitung operator batas dari $\Gamma^{\prime}$, perlu dihitung batas dari anggotaanggota basisnya.

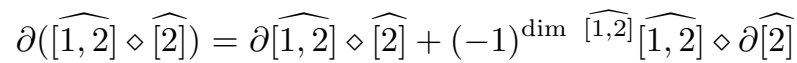

$$
\begin{aligned}
& =(\widehat{[2]}-\widehat{[1]}) \diamond \widehat{[2]}+(-1)^{1} \widehat{[1,2]} \diamond 0 \\
& =(\widehat{[2]} \diamond \widehat{[2]}-\widehat{[1]} \diamond \widehat{[2]})+0 \\
& =\widehat{[2]} \diamond \widehat{[2]}-\widehat{[1]} \diamond \widehat{[2]} \\
& =-\widehat{[1]} \diamond \widehat{[2]})+\widehat{[2]} \diamond \widehat{[2]} \text {. }
\end{aligned}
$$

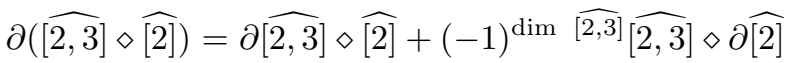

$$
\begin{aligned}
& =(\widehat{[3]}-\widehat{[2]}) \diamond \widehat{[2]}+(-1)^{1} \widehat{[2,3]} \diamond 0 \\
& =(\widehat{[3]} \diamond \widehat{[2]}-\widehat{[2]} \diamond \widehat{[2]})+0 \\
& =\widehat{[3]} \diamond \widehat{[2]}-\widehat{[2]} \diamond \widehat{[2]} \\
& =-\widehat{[2]} \diamond \widehat{[2]})+\widehat{[3]} \diamond \widehat{[2]} \text {. }
\end{aligned}
$$

Selanjutnya tentukan basis dari $Q_{1}$ dan $Q_{2}$

$$
\begin{aligned}
\partial(\widehat{[1,2]} \diamond \widehat{[2]}) & =-\widehat{[1]} \diamond \widehat{[2]})+\widehat{[2]} \diamond \widehat{[2]} \\
& =-1(\widehat{[1]} \diamond \widehat{[2]})+1(\widehat{[2]} \diamond \widehat{[2]}) . \\
\partial(\widehat{[2,3]} \diamond \widehat{[2]}) & =-\widehat{[2]} \diamond \widehat{[2]})+\widehat{[3]} \diamond \widehat{[2]} \\
& =-1(\widehat{[2]} \diamond \widehat{[2]})+1(\widehat{[3]} \diamond \widehat{[2]}),
\end{aligned}
$$

sehingga basis dari $\Gamma^{\prime}$ dapat ditulis dalam bentuk matriks

$$
\partial_{1}=\left[\begin{array}{c}
-1 \\
1
\end{array}\right] .
$$

Untuk memperoleh $Z_{1}\left(\Gamma^{\prime}\right)$, akan dicari ker $\partial_{1}$, dengan menyelesaikan persamaan

$$
\begin{gathered}
{\left[\begin{array}{c}
-1 \\
1
\end{array}\right]\left[\alpha_{1}\right]=\left[\begin{array}{l}
0 \\
0
\end{array}\right] .} \\
{\left[\begin{array}{c}
-\alpha_{1} \\
\alpha_{1}
\end{array}\right]=\left[\begin{array}{l}
0 \\
0
\end{array}\right],}
\end{gathered}
$$

yang memberikan $\alpha_{1}=-\alpha_{1}=0$. Sehingga diperoleh $\alpha_{1}=0$. Karena $Z_{1}\left(\Gamma^{\prime}\right)=0$, $B_{1}\left(\Gamma^{\prime}\right)=0$ akibatnya

$$
H_{1}\left(\Gamma^{\prime}\right) \cong 0 .
$$


Untuk $k=0$,

$$
\widehat{Q}(R)=\left\{\begin{array}{l}
1, R=[1] \times[2] \\
0, \text { selainnya. }
\end{array}\right.
$$

$c([1] \times[2])=\alpha[\widehat{1] \times[2}]([1] \times[2])=\alpha$, dimana $\alpha \in \mathbb{Z}$. Lebih khusus, $Z_{0}(X) \cong$ $C_{0}(X)=\mathbb{Z}$. Oleh karena itu, $H_{0}(X) \cong \mathbb{Z}$.

\section{Kesimpulan}

Misalkan $X$ adalah himpunan kubik. Selanjutnya misalkan $Q \subset X$ adalah kubus dasar. Setiap kubus dasar $Q$ adalah asiklik, yang berarti bahwa

$$
H_{k}(Q) \cong\left\{\begin{array}{l}
\mathbb{Z}, \text { jika } k=0 \\
0, \text { selainnya. }
\end{array}\right.
$$

Namun tidak semua himpunan kubik yang asiklik adalah kubus dasar.

\section{Ucapan Terima kasih}

Penulis mengucapkan terima kasih kepada Bapak Dr. Admi Nazra, Ibu Nova Noliza Bakar, M.Si, Bapak Prof. Dr. Syafrizal Sy, Bapak Prof. Dr. I Made Arnawa dan Bapak Dr. Mahdhivan Syafwan yang telah memberikan masukan dan saran sehingga paper ini dapat diselesaikan dengan baik.

\section{Daftar Pustaka}

[1] Bartle, R.G. dan D.R. Sherbert. 2000.Introduction To Real Analysis, $3^{\text {rd }}$ ed., USA: Copyright Act

[2] Herstein, I. N. 1999. Topics in Algebra. $2^{\text {nd }}$ ed. John Wiley and Sons, New York

[3] Jacob, Bill. 1990. Linear Algebra. W. H. Freeman and Company. New York

[4] Kaczynski. T, K. Mischaikow, M. Mrozek. 2000. Algebraic Topology : A Computational Approach. New York

[5] Kaczynski. T, K. Mischaikow, M. Mrozek. 2004. Computational Homology. Springer-Verlag. New York

[6] Min Yan. 2010. Topology. Hongkong University of Science and Technology. Hongkong 